\title{
El agregado de budesonide inhalatorio a los corticoides orales fue beneficioso luego de una crisis asmática
}

Inhaled budesonide in addition to oral corticosteroids to prevent asthma relapse following discharge from the emergency department. Rowe BH, Bota GW, Fabris L. JAMA 1999; 281: 2119-2126.

\section{Objetivo}

Determinar si la adición de glucocorticoides inhalados (GCl) al tratamiento corticoideo oral, en pacientes externados desde la sala de emergencias luego de una crisis asmática, aporta beneficios adicionales en la reducción de recaídas.

\section{Diseño}

Ensayo clínico aleatorizado, doble ciego y controlado.

Lugar

Servicio de emergencias de un hospital de Canadá.

\section{Pacientes}

De 1006 pacientes de 16 a 60 años, que se presentaron espontáneamente a la guardia (durante los 22 meses que duró el estudio) por crisis asmáticas con un pico flujo $<80 \%$ del esperado, 188 fueron incluidos. El seguimiento fue de 21 días. El principal criterio de exclusión fue tratamiento corticoideo oral o inhalatorio, durante la semana previa.

\section{Intervención}

Todos los pacientes fueron externados con indicación de recibir prednisona $50 \mathrm{mg} /$ día por vía oral, por 7 días. Se aleatorizó a recibir budesonide inhalatorio (Turbuhaler 1600 microgramos/día) o placebo Turbuhaler, por 3 semanas.

\section{Medición de resultados principales}

Se definió como recaída, a tener una o más visitas espontáneas a la guardia ante el empeoramiento de los síntomas. El análisis fue por intención de tratar.

\section{Resultados principales}

De los 188 pacientes, 94 recibieron budesonide inhalatorio y 94 placebo. La tasa de abandono fue del $4 \%$. Luego de 21 días los pacientes con budesonide inhalatorio presentaron en relación al grupo placebo, menor número de recaídas ( $12.8 \%$ vs. $24.5 \%$, RR $0.52, p=0.049$ ). Tuvieron un puntaje algo mejor en el Cuestionario de Calidad de Vida AQLQ (5.9 vs 5.2, $p=0.001$ ); utilizaron menos los agonistas B2 (1.7 inhalaciones menos por día, IC 95\% 0.4 a 3.1, $p=0.01)$. También tuvieron un mejor puntaje de síntomas: menos episodios de tos (5.6 vs $4.7, p=0.004$ ), de disnea ( 5.8 vs $4.9, p=0.001)$, sibilancias (5.9 vs $5, p=0.001)$, despertares nocturnos (6.2 vs 5.4, $\mathrm{p}=0.01$ ); mejoría en la valoración global de su asma (6.2 vs $5.2, p=0.001$ ). No se observó diferencia en la espirometría al cabo de 21 días.

\section{Conclusiones}

En los pacientes asmáticos dados de alta en la guardia con corticoides por vía oral, el agregado de budesonide inhalatorio a altas dosis fue beneficioso.

\section{Comentario}

Entre los pacientes asmáticos externados desde la sala de emergencias luego de una crisis aguda aparentemente bien controlada, el $17 \%$ presenta una recaída dentro de los 14 días posteriores. 1 Es importante destacar que aún cuando los síntomas hayan desaparecido y la función pulmonar mejorado, la crisis no ha sido resuelta completamente. La obstrucción al flujo aéreo puede persistir por varios días, no existiendo una medida objetiva, como el

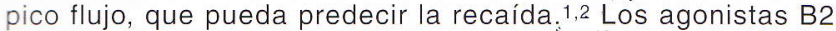
inhalatorios y los corticoides sistémicos (CS) son el tratamiento de primera línea ante una crisis asmática. Existe fuerte evidencia a favor del uso de CS durante los 5 a 10 días posteriores a la externación, 3,4 en tanto que la recomendación para el uso de GCl es inconsistente. 5

Este estudio demuestra que la adición de altas dosis de $\mathrm{GCl}$ al tratamiento corticoideo oral luego de una crisis asmática moderada a severa, disminuye el número de recaídas y mejora la ćalidad de vida, durante el período evaluado. Se observó una reducción del $50 \%$ en la incidencia de recaídas $(R R R=50 \%)$, evidenciándose que por cada 9 pacientes tratados 1 recaída podría prevenirse. Es de notar que no se encontraron diferencias significativas en los valores espirométricos.

Este estudio estuvo bien realizado. Tuvo un alto nivel de seguimien- to y la tasa de abandono fue del $4 \%$. Las drogas en ambos grupos fueron bien toleradas, con una adherencia al tratamiento del $80 \%$.

Otros dos ensayos que analizaron el uso de GCl más CS al alta, fueron reunidos en una revisión sistemática junto a este estudio. A diferencia de este estudio, los resultados finales no demostraron un beneficio estadísticamente significativo en el outcome primario, a pesar de una tendencia a favor del tratamiento con GCl. Esto probablemente se deba a que los otros estudios utilizaron dosis moderadas de $\mathrm{GCl}$; pero sí coincidieron en la falta de modificación de la función pulmonar, resultado que se correlaciona con los obtenidos en estudios previos, donde se analizó el uso de GC más CS durante la permanencia en guardia. ${ }^{6}$ Por lo tanto nuevos estudios se requieren para confirmar el beneficio de los $\mathrm{GCl}$ en este período. A su vez es necesario determinar la dosis óptima y duración del tratamiento, como también su utilidad en niños.

La consulta a un servicio de emergencias es indicador de un mal control ambulatorio o de una inadecuada instrucción personalizada en el manejo de las exacerbaciones. ${ }^{4}$ El momento de la externación luego de una crisis aguda puede ser considerada como una buena oportunidad para iniciar al paciente en el uso de $\mathrm{GCl}$ y para reforzar otras medidas educacionales.

\section{Dra. María Silvina Gaglio [ Ex residente de Medicina Interna. Hospital Ramos Mejía. ]}

\footnotetext{
Referencias

1. Emerman C, Woodruff P, Cydulka R,

temerman C, Woodruff P, Cydulka R, C

2. NAEPP Expert Panel Report II: Pharmacology therapy: managing exacerbations of asthma. 2000 Up To Date, vol 8, № 2

3. Chapman K, MD; Verbeek P, MD, White J, Rebuck A, MD. Efect of a short course of prednisone in the prevention of early relapse after the emergency room treatment of acute asthma. NeJM 1991; 324: 788-94.

4. O Driscoll B, Kaltca S, Wilson M, Pickening C, Carroll K, Woodcock A. Double-blind trial of steroid tapering in acute asthma. LANCET 1993; 341: 324-27.

5. Jinich P. Asma. PROFAM 1997.

E. Edmonds ML, Camargo CA, Saunders LD, Brenner BE, Rowe BH. Inhaled steroids in acute asthma following emergency department discharge. The Cochrane Library, Issue 3, 2000.
} 